\title{
SOME NEW CLASSES OF KERNELS WHOSE FREDHOLM DETERMINANTS HAVE ORDER LESS THAN ONE
}

\author{
BY
}

DALE W. SWANN

\begin{abstract}
Let $K(s, t)$ be a complex-valued $L_{2}$ kernel on the square $a \leqq s, t \leqq b$ and $\left\{\lambda_{v}\right\}$, perhaps empty, denote the set of finite characteristic values (f.c.v.) of $K$, arranged according to increasing modulus. Such f.c.v. are complex numbers appearing in the integral equation $\phi_{v}(s)=\lambda_{v} \int_{a}^{b} K(s, t) \phi_{v}(t) d t$, where the $\phi_{v}(s)$ are nontrivial $L_{2}$ functions on $[a, b]$. Further let $k_{1}=\int_{a}^{b} K(s, s) d s$ be well defined so that the Fredholm determinant of $K, D(\lambda)$, exists, and let $\mu$ be the order of this entire function. It is shown that (1) if $K(s, t)$ is a function of bounded variation in the sense of Hardy-Krause, then $\mu \leqq 1 ;(2)$ if in addition to the assumption (1), $K(s, t)$ satisfies a uniform Lipschitz condition of order $\alpha>0$ with respect to either variable, then $\mu<1$ and $k_{1}=\sum_{v} 1 / \lambda_{v}$; (3) if $K(s, t)$ is absolutely continuous as a function of two variables and $\partial^{2} K / \partial s \partial t$ (which exists almost everywhere) belongs to class $L_{p}$ for some $p>1$, then $\mu<1$ and $k_{1}=\sum_{v} 1 / \lambda_{v}$. In (2) and (3), the condition $k_{1} \neq 0$ implies $K(s, t)$ possesses at least one f.c.v.
\end{abstract}

1. Introduction. Let $K(s, t)$ be a complex-valued $L_{2}$ kernel on the square $a \leqq s, t \leqq b$, by which we mean $\int_{a}^{b} \int_{a}^{b}|K(s, t)|^{2} d s d t<\infty$, and let $\left\{\lambda_{v}\right\}$, perhaps empty, denote the set of finite characteristic values (f.c.v.) of $K$, arranged according to increasing modulus. Such f.c.v. are complex numbers satisfying

$$
\phi_{v}(s)=\lambda_{v} \int_{a}^{b} K(s, t) \phi_{v}(t) d t,
$$

where the $\phi_{v}(s)$ are nontrivial $L_{2}$ functions on $[a, b]$. If $K$ does not possess additional structure or fall into certain specialized categories (e.g., Volterra, normal, degenerate, positive definite) the number of such f.c.v., at most countable, is generally unknown.

Briefly stated, in this paper we show that if $K(s, t)$ belongs to certain classes of functions of importance in the study of double Fourier series (and hence of physical as well as mathematical interest), then $\sum_{v=1}^{\infty} 1 /\left|\lambda_{v}\right|<\infty$. For such cases, if $\sum_{v=1}^{\infty} 1 / \lambda_{v} \neq 0$, a condition easily ascertained as shown below, then $K(s, t)$ will have at least one f.c.v.

Received by the editors November 18, 1970.

AMS 1970 subject classifications. Primary 45B05, 45H05; Secondary 26A45, $26 \mathrm{~A} 69$.

Key words and phrases. $L_{2}$ kernels, characteristic values, integral equations, Fredholm determinant, Lipschitz (Hölder) conditions, functions of bounded variation in Hardy-Krause sense, absolutely continuous functions of two variables.

Copyright (C) 1971, American Mathematical Society 
To be more explicit, we shall assume here (in order to avoid essentially pathological situations) that $K(s, s)$ is both measurable and summable so that trace $K$ $=k_{1}=\int_{a}^{b} K(s, s) d s$ is well defined. Consequently the Fredholm determinant of $K$ exists for such kernels and may be expressed as

$$
D(\lambda)=\exp \left(-k_{1} \lambda\right) \prod_{v=1}^{\infty}\left(1-\lambda / \lambda_{v}\right) \exp \left(\lambda / \lambda_{v}\right)=1+\sum_{v=1}^{\infty} d_{v} \lambda^{v}
$$

where for $\nu \geqq 1$

$$
d_{v}=\frac{(-1)^{v}}{\nu !} \int_{a}^{b} \cdots \int_{a}^{b}\left|\begin{array}{cccc}
K\left(s_{1}, s_{1}\right) & K\left(s_{1}, s_{2}\right) & \cdots & K\left(s_{1}, s_{v}\right) \\
K\left(s_{2}, s_{1}\right) & K\left(s_{2}, s_{2}\right) & \cdots & K\left(s_{2}, s_{v}\right) \\
\vdots & \vdots & & \vdots \\
K\left(s_{v}, s_{1}\right) & K\left(s_{v}, s_{2}\right) & \cdots & K\left(s_{v}, s_{v}\right)
\end{array}\right| d s_{1} d s_{2} \cdots d s_{v}
$$

The order $\mu$ of this entire function $D(\lambda)$, at most $2\left(^{1}\right)$, is given by

$$
\mu=\limsup _{\nu \rightarrow \infty} \frac{\nu \log \nu}{\log \left(1 /\left|d_{v}\right|\right)} .
$$

If $\mu \neq 0,1, K$ obviously possesses an infinite number of f.c.v.

In [3] we characterized, without reference to their analytic structure, those kernels with only a finite number of f.c.v. In this paper, we impose conditions on the structure of $K(s, t)$ as a function of the two real variables $s$ and $t$ which permit us to conclude that $\mu<1$, so that $\sum_{v=1}^{\infty} 1 /\left|\lambda_{v}\right|<\infty$, and $D(\lambda)$ assumes the form

$$
D(\lambda)=\prod_{v=1}^{\infty}\left(1-\lambda / \lambda_{v}\right), \quad \text { with } k_{1}=\sum_{v=1}^{\infty} 1 / \lambda_{v}
$$

For such kernels, $k_{1} \neq 0$ is then a sufficient condition that there be at least one f.c.v.

Fredholm himself [1] (see also Lalesco [4], [5], and Cochran [6]) showed that if $K(s, t)$ satisfies a uniform Lipschitz condition of order $\alpha, 0<\alpha \leqq 1$ (frequently called Hölder continuity with exponent $\alpha$ ), in either $s$ or $t$, that is, e.g.,

$$
\left|K\left(s, t_{2}\right)-K\left(s, t_{1}\right)\right| \leqq A\left|t_{2}-t_{1}\right|^{\alpha}, \quad t_{2}, t_{1} \text { in }[a, b],
$$

where $A$ is a constant, then $\mu \leqq 1 /\left(\alpha+\frac{1}{2}\right)$. If $\alpha>\frac{1}{2}$, therefore, $\mu<1$, and the representation (5) is valid $\left({ }^{2}\right)$.

In order to extend results of the above type to wider classes of kernels, we introduce in $\$ 2$ functions of two variables of bounded variation, concentrating on those satisfying the definition of Hardy-Krause [8], as well as functions of two

(1) A fact established by Fredholm [1] for bounded, integrable kernels and by Carleman [2] for general $L_{2}$ kernels.

$\left({ }^{2}\right)$ Note that $\alpha>1$ in (6) implies $K(s, t)$ is a function of $s$ alone; the representation (5) then holds automatically. 
variables which are absolutely continuous. The interplay between such classes of functions and those satisfying uniform Lipschitz conditions is sketched. In $\S 3$, we use a variant of Fredholm's method to estimate the magnitude of the coefficients $d_{v}$ of (3) to prove the following for $K(s, t)$ defined on the square $S: a \leqq s, t \leqq b$.

THEOREM 1. If $K(s, t)$ is a function of bounded variation in the sense of HardyKrause, then $\mu \leqq 1$.

THEOREM 2. If in addition to the assumption of Theorem $1, K(s, t)$ also satisfies a uniform Lipschitz condition of order $\alpha>0$, then $\mu<1$ and $D(\lambda)$ has the representation (5).

THEOREM 3. If $K(s, t)$ is absolutely continuous and $\partial^{2} K / \partial s \partial t$ (which exists almost everywhere) belongs to class $L_{p}$ for some $p>1$, then $D(\lambda)$ may likewise be represented as in $(5)\left({ }^{3}\right)$.

2. Functions of bounded variation; absolute continuity. Functions $g(s)$ of bounded variation (hereafter referred to as BV) of one variable defined for simplicity on $s_{0} \leqq s \leqq s_{1}$ are of great interest and usefulness because of their valuable properties. Such properties, particularly with respect to additivity, decomposability into monotone functions, continuity, differentiability, measurability, integrability, and so on, have been much studied.

It is largely to the possession of these properties that functions of BV owe their important role in the study of rectifiable curves, Fourier and other series, Stieltjes and other integrals, and the calculus of variations. We recall that a complexvalued function of a real variable, $g(s)=g_{1}(s)+i g_{2}(s)$, is said to be of BV if and only if $g_{1}(s)$ and $g_{2}(s)$ are real-valued functions of BV.

When we come to the question of extending the definition of functions of BV to functions of two or more variables, we can proceed in many ways. Proposers of definitions of $\mathrm{BV}$ for functions $f(s, t)$ have been actuated mainly by the desire to single out for attention a class of functions having properties analogous to some particular properties of a function $g(s)$ of $\mathrm{BV}$. It has long since become apparent that to preserve properties of one sort the definition of BV for $g(s)$ should be extended to $f(s, t)$ in one way, while to preserve properties of another sort a quite different extension may be needed. For further elucidation on this point, reference is made to the two papers of Adams and Clarkson [9], [10] where seven different definitions of functions $f(s, t)$ of $\mathrm{BV}$ are introduced and the properties of the functions in the seven classes are compared.

In this paper we choose to utilize a definition of functions $f(s, t)$ of $\mathrm{BV}$ due to Hardy and Krause [8], [9], [10] and we shall say that $f(s, t)$ belongs to class $H$ if $f(s, t)$ satisfies that definition. As for functions of one variable, we agree that

$\left({ }^{3}\right)$ Conditions under which the theorems are valid can be lightened to some extent. This question is touched on briefly. 
$f(s, t)=f_{1}(s, t)+i f_{2}(s, t) \in H$ if and only if $f_{i}(s, t), i=1,2 \in H$, where $f_{i}(s, t)$ is real valued.

Definitions. The function $f(s, t)$ is assumed to be finitely defined in a rectangle $R: a \leqq s \leqq b, c \leqq t \leqq d$. By the term net we shall, unless otherwise specified, mean a set of parallels to the axes:

$$
\begin{array}{lll}
s=s_{i} & (i=0,1,2, \ldots, m), & a=s_{0}<s_{1}<s_{2}<\cdots<s_{m}=b, \\
t=t_{j} & (j=0,1,2, \ldots, n), & c=t_{0}<t_{1}<t_{2}<\cdots<t_{n}=d .
\end{array}
$$

Each of the smaller rectangles into which $R$ is divided by a net will be called a cell. We employ the notation

$$
\begin{aligned}
\Delta_{10} f\left(s_{i}, t_{j}\right) & =f\left(s_{i+1}, t_{j}\right)-f\left(s_{i}, t_{j}\right), \\
\Delta_{01} f\left(s_{i}, t_{j}\right) & =f\left(s_{i}, t_{j+1}\right)-f\left(s_{i}, t_{j}\right), \\
\Delta_{11} f\left(s_{i}, t_{j}\right) & =\Delta_{10}\left(\Delta_{01} f\left(s_{i}, t_{j}\right)\right) \\
& =f\left(s_{i+1}, t_{j+1}\right)-f\left(s_{i+1}, t_{j}\right)-f\left(s_{i}, t_{j+1}\right)+f\left(s_{i}, t_{j}\right) .
\end{aligned}
$$

The function $f(s, t)$ is said to be of BV in the sense of Hardy and Krause, i.e., $f(s, t) \in H$, if (a) the sum

$$
\sum_{i=0, j=0}^{m-1, n-1}\left|\Delta_{11} f\left(s_{i}, t_{j}\right)\right|
$$

is bounded for all nets and in addition (b) $f(\bar{s}, t)$ is of $\mathrm{BV}$ in $t$ for at least one $\bar{s}$ and $f(s, \bar{t})$ is of BV in $s$ for at least one $\bar{t}$.

Relevant properties of $f(s, t) \in H$ are:

$\mathrm{H}-1$. If $f(s, t) \in H, f(\bar{s}, t)$ is of BV in $t$ for every $\bar{s}$, and $f(s, \bar{t})$ is of $\mathrm{BV}$ in $s$ for every $\bar{t}$.

H-2. If $f(s, t) \in H,|f(s, t)|$ is bounded in $R$.

$\mathrm{H}-3$. The class $H$ is closed under addition and subtraction.

$\mathrm{H}-4$. The class $H$ is closed under multiplication.

H-5. The class $H$ is closed under division if the denominator is bounded away from zero.

H-6. If $f(s, t) \in H$ on rectangles $R_{1}$ and $R_{2}$ and if $R=R_{1} \cup R_{2}$ is a rectangle, then $f(s, t) \in H$ on $R$.

H-7. If $f(s, t) \in H$ on a rectangle $R$ and if $R_{1} \subset R$ is a rectangle, then $f(s, t) \in H$ on the subrectangle $R_{1}$.

H-8. A necessary and sufficient condition that $f(s, t)=f_{1}(s, t)+i f_{2}(s, t)$ belong to $H$ is that the real and imaginary parts of $f(s, t)$ each be expressible as the difference between pairs of bounded functions $f_{11}(s, t), f_{12}(s, t)$ and $f_{21}(s, t)$, $f_{22}(s, t)$, i.e., $f_{1}(s, t)=f_{11}(s, t)-f_{12}(s, t), f_{2}(s, t)=f_{21}(s, t)-f_{22}(s, t)$, where the $f_{i j}(s, t)$ are called "quasi-monotone" and satisfy $\Delta_{10} f_{i j}(s, t) \geqq 0, \Delta_{01} f_{i j}(s, t) \geqq 0$, $\Delta_{11} f_{i j}(s, t) \geqq 0, i, j=1,2$. (Increments in $s$ and $t$ are supposed positive.) 
H-9. If $f(s, t) \in H$, the discontinuities of $f(s, t)$ are located on a denumerable number of parallels to the axes, i.e., $f(s, t)$ is continuous in $(s, t)$ almost everywhere, (a.e.).

$\mathrm{H}-10$. The double Riemann integral over $R$ of a function $f(s, t) \in H$ always exists.

H-11. If $f(s, t) \in H$ then $\partial^{2} f / \partial s \partial t$ exists a.e. in $R$.

H-12. $f(s, t)$ is called factorable if and only if in $R$ we have $f(s, t) \equiv g(s) h(t)$, with neither $g$ nor $h$ identically zero. Then $\Delta_{11} f(s, t)=\Delta g(s) \Delta h(t)$ and a necessary and sufficient condition that a factorable function belong to class $H$ is that each factor be of BV. Note that a factorable $f(s, t) \in H$ functioning as the kernel of an integral equation is automatically a degenerate kernel.

It is perhaps worth noting that the definition of a function $f(s, t)$ of $\mathrm{BV}$ in the sense of Hardy and Krause is "the most restrictive" of any of the seven given by Adams and Clarkson. The functions of class $H$ are those which it is convenient to consider in the study of double Fourier series.

Another important class of functions which plays a prominent part in the Lebesgue theory of integration and differentiation (for functions of one variable) is the class of absolutely continuous (AC) functions. We recall that a necessary and sufficient condition that a function $F(s)$, defined on an interval, may be an indefinite integral of a summable function is that it should be AC. Extension of the AC concept to functions of two variables is straightforward [8].

Definition. If a function $f(s, t)$, defined in a rectangle $a \leqq s \leqq b, c \leqq t \leqq d$, is such that, for any finite or infinite set of nonoverlapping cells $\sigma$ (any two of which may have portions of their boundaries in common), the sum

$$
\sum_{\sigma}\left|\Delta_{11} f(s, t)\right|
$$

is less than an arbitrarily fixed positive number $\eta$, for all sets of such cells which satisfy the condition that the sum of their measures is less than some positive number $\varepsilon_{\eta}$, the function $f(s, t)$ is said to be AC in the rectangle $R$.

The characteristic properties of functions of one variable which are AC are also possessed by AC functions of several variables; viz., $f(s, t) \in \mathrm{AC}$ implies $f(s, t)$ is continuous, $f(s, t)$ is of bounded variation (more specifically $f(s, t) \in H)$, etc. All these properties follow from two fundamental relationships we quote below. Proofs can be found in [8].

AC-1. The two-dimensional analogue of the property quoted above for AC functions of one variable holds; viz., a necessary and sufficient condition that a function of two variables, defined in a cell $\Delta$, should be the indefinite integral of some function, summable in the cell, is that it should be AC in the cell.

AC-2. The indefinite integral

$$
f(s, t)=\int_{a}^{s} \int_{c}^{t} g\left(s_{1}, t_{1}\right) d s_{1} d t_{1}
$$

where $g(s, t)$ is summable on $R$ has the property that $\partial^{2} f / \partial s \partial t$ exists a.e. in $R$ and $\partial^{2} f / \partial s \partial t=\partial^{2} f / \partial t \partial s=g(s, t)$ a.e. in $R$. 
We have already mentioned in $\S 1$ the class of functions of two variables satisfying uniform Lipschitz conditions of order $\alpha$ in one variable or the other; such will be referred to hereafter as of class $L(\alpha), 0<\alpha \leqq 1$. Functions of one variable belonging to $L(1)$ are also of $\mathrm{BV}$; on the other hand there are functions of one variable of $\mathrm{BV}$ which satisfy no Lipschitz condition and functions belonging to $L(\alpha)$ with $\alpha<1$ exist which have unbounded variation [11]. Similar remarks apply to functions $f(s, t) \in L(\alpha)$; they need not be continuous, of $\mathrm{BV}$, or even measurable.

\section{Order of $D(\lambda)$ for various classes of functions.}

THEOREM 1. On the square $S: a \leqq s, t \leqq b$, let the $L_{2}$ kernel $K(s, t)$ belong to the class $H$ (functions of $B V$ in the sense of Hardy-Krause). Then $\mu$, the order of its Fredholm determinant $D(\lambda)$, satisfies $\mu \leqq 1$.

Proof of Theorem 1. Consider (3) in which the coefficients $d_{v}$ must be estimated in order to discover the order of $D(\lambda)$. To condense the notation, define

$$
A_{v}=\left|\begin{array}{cccc}
K\left(s_{1}, s_{1}\right) & K\left(s_{1}, s_{2}\right) & \cdots & K\left(s_{1}, s_{v}\right) \\
K\left(s_{2}, s_{1}\right) & K\left(s_{2}, s_{2}\right) & \cdots & K\left(s_{2}, s_{v}\right) \\
\vdots & \vdots & & \vdots \\
K\left(s_{v}, s_{1}\right) & K\left(s_{v}, s_{2}\right) & \cdots & K\left(s_{v}, s_{v}\right)
\end{array}\right|
$$

To begin, we modify $A_{v}$ in accordance with the laws for carrying out operations on determinants, but in such a way that the value of $A_{v}$ does not change. First we subtract the second column of $A_{v}$ from the first column, the third column from the second, ..., and finally the $\nu$ th column from the $(\nu-1)$ th, obtaining

$$
A_{v}=\left|\begin{array}{ccccc}
K\left(s_{1}, s_{1}\right)-K\left(s_{1}, s_{2}\right) & K\left(s_{1}, s_{2}\right)-K\left(s_{1}, s_{3}\right) & \cdots & K\left(s_{1}, s_{v-1}\right)-K\left(s_{1}, s_{v}\right) & K\left(s_{1}, s_{v}\right) \\
K\left(s_{2}, s_{1}\right)-K\left(s_{2}, s_{2}\right) & K\left(s_{2}, s_{2}\right)-K\left(s_{2}, s_{3}\right) & \cdots & K\left(s_{2}, s_{v-1}\right)-K\left(s_{2}, s_{v}\right) & K\left(s_{2}, s_{v}\right) \\
\vdots & \vdots & & \vdots & \vdots \\
K\left(s_{v}, s_{1}\right)-K\left(s_{v}, s_{2}\right) & K\left(s_{v}, s_{2}\right)-K\left(s_{v}, s_{3}\right) & \cdots & K\left(s_{v}, s_{v-1}\right)-K\left(s_{v}, s_{v}\right) & K\left(s_{v}, s_{v}\right)
\end{array}\right| .
$$

Now perform a second series of operations on $A_{v}$ in (9) by subtracting the second row from the first row, the third row from the second, .., the $\nu$ th row from the $(\nu-1)$ th. The value of $A_{v}$ is not altered by this series of operations. The resulting form of $A_{v}$ is now unwieldy to write in display form, so we shall only specify its elements which we denote by $a_{i j}$ :

$$
\begin{array}{ll}
a_{i j}=\Delta_{11} K\left(s_{i}, s_{j}\right), & i, j=1,2, \ldots, v-1, \\
a_{i v}=-\Delta_{10} K\left(s_{i}, s_{v}\right), & i=1,2, \ldots, \nu-1, \\
a_{v j}=-\Delta_{01} K\left(s_{v}, s_{j}\right), & j=1,2, \ldots, \nu-1, \\
a_{v v}=K\left(s_{v}, s_{v}\right), &
\end{array}
$$

where we have used the definitions (7). 
Let the cofactor of an element $a_{i j}$ be $A_{i j}$, so that in general we have for the evaluation of $A_{v}$ :

$$
A_{v}=\sum_{j=1}^{\nu} a_{i j} A_{i j}=\sum_{i=1}^{\nu} a_{i j} A_{i j} .
$$

In particular we may expand $A_{v}$ by the $\nu$ th column so that $A_{v}=\sum_{i=1}^{v} a_{i v} A_{i v}$ and we may bound the absolute value of $A_{v}$ by

$$
\left|A_{v}\right| \leqq \sum_{i=1}^{v}\left|a_{i v}\right|\left|A_{i v}\right| \leqq B \sum_{i=1}^{v}\left|A_{i v}\right|,
$$

where $B$ is a constant, since by property $\mathrm{H}-2, K(s, t) \in H$ is bounded on $S$.

Next we estimate $\left|A_{i v}\right|$, where $A_{i v}$ is the $(\nu-1) \times(\nu-1)$ determinant obtained from $A_{v}$ by striking out its $i$ th row and $\nu$ th column. To carry out this estimation we utilize the Hadamard inequality [12]: if $B$ is an $n \times n$ matrix with elements $b_{h k}$ then

$$
|\operatorname{det} B|^{2} \leqq \prod_{n=1}^{n} \sum_{k=1}^{n}\left|b_{h k}\right|^{2}=\prod_{n=1}^{n} \sum_{k=1}^{n}\left|b_{k h}\right|^{2},
$$

the last equality holding because $B$ and its transpose have the same determinant.

If we now apply (12) to estimate our determinant $A_{i v}$ we obtain

$$
\left|A_{i v}\right|^{2} \leqq\left.\left.\prod_{j=1}^{v-1}\left\{\sum_{k=1}^{i-1}\left|a_{k j}\right|^{2}+\sum_{k=i+1}^{v}\left|a_{k j}\right|^{2}\right\} \equiv \prod_{j=1}^{v-1} \sum_{k=1}^{v}\right|_{k j}\right|^{2},
$$

where the prime on the summation in (13) indicates the term corresponding to $k=i$ is missing. Referring to (10) and (7) we see that $\left|a_{k j}\right|^{2} \leqq C\left|a_{k j}\right|$, where $C$ is some constant, while

$$
\sum_{k=1}^{v}\left|a_{k j}\right| \leqq\left|\Delta_{01} K\left(s_{v}, s_{j}\right)\right|+\sum_{k=1}^{v-1}\left|\Delta_{11} K\left(s_{k}, s_{j}\right)\right|, \quad 1 \leqq j \leqq \nu-1,
$$

and the right-hand side of (14) is uniformly bounded (independent of the magnitude of $\nu$ ) because $K(s, t) \in H$. Thus (13) becomes

$$
\left|A_{i v}\right| \leqq C^{\nu-1},
$$

where $C$ is some (generic) constant not necessarily the same each time it appears. Combining (15) with (11), (8), and (3) we find

$$
\left|d_{v}\right| \leqq C^{\nu-1} /(\nu-1) !
$$

Using Stirling's expansion [13] for the factorial function, viz., $(\nu-1)$ ! $=e^{-v} \nu^{\nu-1 / 2} \sqrt{ }(2 \pi)\left[1+1 / 12 \nu+O\left(1 / \nu^{2}\right)\right]$ as $\nu \rightarrow \infty$, we then have, for large $\nu, 1 /\left|d_{v}\right|$ $\geqq C^{\nu-1} \nu^{\nu-1 / 2}$, from which it follows readily by (4) that $\mu \leqq 1$, as was to be proved.

THEOREM 2. If in addition to the assumption of Theorem $1, K(s, t) \in L(\alpha), \alpha>0$, then $\mu<1$ and $D(\lambda)$ has the representation (5). 
Proof of Theorem 2. The proof proceeds along the lines of Theorem 1; in estimating the sum in (13), however, we now have

$$
\left|a_{k j}\right|^{2} \leqq C\left|s_{j+1}-s_{j}\right|^{\alpha} \cdot\left|a_{k j}\right|, \quad 1 \leqq j \leqq \nu-1,
$$

since $K(s, t) \in L(\alpha)$. It follows that

$$
\left|A_{v}\right| \leqq \nu C^{\nu-1} \prod_{j=1}^{\nu-1}\left|s_{j+1}-s_{j}\right|^{\alpha / 2},
$$

and since the determinant $A_{v}(8)$ is a symmetric function of the arguments $s_{1}, s_{2}, \ldots, s_{v}$, there is no loss of generality in assuming that $a \leqq s_{1} \leqq s_{2} \leqq \cdots \leqq s_{v} \leqq b$. A classical argument [1] shows the right-hand side of (17) is maximized if the $s_{j}$ are spaced uniformly on the segment $b-a$. Hence

$$
1 /\left|d_{\nu}\right| \geqq C^{\nu-1}(\nu-1) !(\nu-1)^{\alpha(\nu-1) / 2},
$$

and using once again the Stirling formula and (4), we conclude $\mu \leqq 2 /(2+\alpha)<1$, as was to be proved.

It is worth noting here that Fredholm's result for kernels of class $L(\alpha), \alpha>\frac{1}{2}$, mentioned in $\S 1$ can be proved with only slightly more effort under the weaker condition $\left|K\left(s, t_{2}\right)-K\left(s, t_{1}\right)\right| \leqq A(s)\left|t_{2}-t_{1}\right|^{\alpha}$, where $A(s)$ is nonnegative and summable $\left({ }^{4}\right)$. Consequently, our Theorem 1 can be established under the somewhat weaker condition that

$$
\sum_{j=1}^{v-1}\left|K\left(s, t_{j+1}\right)-K\left(s, t_{j}\right)\right|=\sum_{j=1}^{v-1}\left|\Delta_{01} K\left(s, t_{j}\right)\right| \leqq B(s),
$$

where $B(s)$ is nonnegative and summable. In the former case, we might say $K(s, t)$ satisfies a relatively uniform Lipschitz condition with $\alpha>\frac{1}{2}$; in the latter that $K(s, t)$ is relatively uniformly of $\mathrm{BV}$. In these terms, our Theorem 2 can be proved if $K(s, t)$ is relatively uniformly of $\mathrm{BV}$ in one variable and satisfies a relatively uniform Lipschitz condition of order $\alpha>0$ in the same or the other variable. The precise relationship between the various classes of functions and the degree of lightening of the conditions of validity for Theorems 1,2 is not entirely clear, however.

THEOREM 3. On the square $S: a \leqq s, t \leqq b$, let $K(s, t) \in A C$ and assume that $\partial^{2} K / \partial s \partial t$ (which exists a.e. by property AC-2) $\in L_{p}$ for some $p>1$. Then $D(\lambda)$ may likewise be represented as in (5).

Proof of Theorem 3. We shall make our proof of this theorem depend upon Theorem 2. We know $K(s, t) \in H$; we shall show $K(s, t) \in L(1 / q)$ where $q$ is the Lebesgue index conjugate to $p$, i.e., $1 / p+1 / q=1 ; p, q>1$.

(4) These observations are due to Cochran [7]. 
By property AC-1, there exists a summable function $k(s, t)$ on $S$ such that

$$
K(s, t)=\int_{a}^{s} \int_{a}^{t} k(u, v) d u d v
$$

and by AC-2, $\partial^{2} K / \partial s \partial t=\partial^{2} K / \partial t \partial s=k(s, t)$ a.e. on $S$. For $(s, t)$ and $(s, t+h) \in S$,

$$
|K(s, t+h)-K(s, t)| \leqq \int_{a}^{s} \int_{t}^{t+h}|k(u, v)| d u d v
$$

Since $k(u, v) \in L_{p}, p>1$, Hölder's inequality in its two-dimensional form [12] yields

$$
\begin{aligned}
|K(s, t+h)-K(s, t)| & \leqq\left\{\int_{a}^{s} \int_{t}^{t+h}|k(u, v)|^{p} d u d v\right\}^{1 / p} \cdot\left\{\int_{a}^{s} \int_{t}^{t+h} 1^{a} d u d v\right\}^{1 / q} \\
& \leqq\left\{\int_{a}^{b} \int_{a}^{b}|k(u, v)|^{p} d u d v\right\}^{1 / p}(b-a)^{1 / a} h^{1 / q} \\
& =A h^{1 / q}
\end{aligned}
$$

independent of $s$. Thus $K(s, t) \in L(1 / q)$ with $q>1$, so Theorem 2 applies and the desired result ensues.

\section{REFERENCES}

1. I. Fredholm, Sur une classe d'équations fonctionnelles, Acta Math. 27 (1903), 365-390.

2. T. Carleman, Zur Theorie der linearen Integralgleichungen, Math. Z. 9 (1921), 196-217.

3. D. W. Swann, Kernels with only a finite number of characteristic values, Proc. Cambridge Philos. Soc. (to appear).

4. T. Lalesco, Sur l'ordre de la fonction entière $D(\lambda)$ de Fredholm, C. R. Acad. Sci. Paris 145 (1907), 906-907.

5. - Introduction à la theorie des équations intégrales, Hermann, Paris, 1912, pp. 86-89.

6. J. A. Cochran, The existence of eigenvalues for the integral equations of laser theory, Bell System Tech. J. 44 (1965), 77-88. MR 30 \#1368.

7. - The analysis of linear integral equations, McGraw-Hill, New York, 1972.

8. E. W. Hobson, The theory of functions of a real variable and the theory of Fourier's series. Vol. 1, Dover, New York, 1958. MR 19, 1166.

9. J. A. Clarkson and C. R. Adams, On definitions of bounded variation for functions of two variables, Trans. Amer. Math. Soc. 35 (1933), 824-854.

10. - Properties of functions $f(x, y)$ of bounded variation, Trans. Amer. Math. Soc. 36 (1934), 711-730.

11. I. P. Natanson, Theory of functions of a real variable, GITTL, Moscow, 1950; English transl., Ungar, New York, 1955. MR 12, 598; MR 16, 804.

12. E. F. Beckenbach and R. Bellman, Inequalities, Ergebnisse der Mathematik und ihrer Grenzgebiete, Neue Folge, Band 30, Springer-Verlag, New York, 1965, p. 21. MR 33 \#236.

13. E. T. Copson, An introduction to the theory of functions of a complex variable, Clarendon Press, Oxford, 1935, p. 223.

Bell Telephone laboratories, Inc., WhipPANY, New JeRSEY 07981 